\title{
EDITORIAL
}

\section{La genómica: el nuevo horizonte de la medicina}

\author{
Genomics: the new horizon in clinical medicine \\ José Félix Patiño Restrepo, MD, FACS (Hon), MACC (Hon) \\ Editor, Revista Colombiana de Cirugía
}

A mediados del siglo XIX, el monje agustiniano austriaco Gregor Johann Mendel (I822-I884), trabajando con plantas del jardín del monasterio, descubrió los principios fundamentales de la herencia y con ello se han desarrollado la genética* y la genómica**. Es uno de los hitos de mayor trascendencia en el devenir histórico de la biología y de la biomedicina.

Laborando en los Laboratorios Cavendish de la Universidad de Cambridge, el estadounidense James D. Watson (n. 1928), y los británicos Francis H. C. Crick (1916-2004) y Maurice H. F. Wilkins (I9I6-2004), con el valioso aporte de Rosalind Franklin (1920-I958) con sus imágenes de difracción con rayos $\mathrm{X}$, descubrieron la estructura molecular del ácido desoxirribonucleico (ADN). La publicación original apareció en $\mathrm{Na}$ ture el 25 de abril de i953. Con ello, se abrió un esplendoroso panorama porque, al estudiar el reduccionismo las bases moleculares de la vida y la enfermedad, la medicina, que hasta entonces era un arte o una ciencia aplicada, ahora sí se convirtió en una verdadera ciencia, la biomedicina, "la más joven de las ciencias", como la ha llamado Lewis Thomas (1913-1993), quien fue decano de medicina de la Universidad de Yale. En octubre de 1962, Watson, Crick y Wilkins recibieron el Premio Nobel. El nombre de Rosalind Franklin no figuró porque el Premio Nobel no se adjudica a personas ya fallecidas.

Con el descubrimiento de la estructura molecular en forma de doble hélice del ADN, nació el nuevo paradigma, la biomedicina. Ahora se pudieron conocer en el interior de la célula los procesos moleculares de la vida y la enfermedad, y en la medicina se abrió un panorama deslumbrante que ha venido creciendo en forma rápida y que transformó totalmente tanto la medicina como la atención de la salud.

"This figure is purely diagrammatic. The two ribbons symbolize the two phosphate-sugar chains, and the horizontal rods the pairs of bases holding the chains together. The vertical line marks the fibre axis".

Palabras clave: genómica; genética; historia; investigación en medicina traslacional.

* Diccionario Académico de la Medicina: genética: (Del griego gennetikós, 'que genera') f. Otras ciencias biomédicas. Parte de la biología que trata de la herencia y de lo relacionado con ella.

** DRAE genómica: 2. f. Biol. Estudio de la composición, estructura y función del genoma.

Fecha de recibido: 10/01/2018 Fecha de aceptado: 11/01/2018

Correspondencia: doctor José Félix Patiño Restrepo/ Correo electrónico: jfpatinore@gmail.com

Citar como: Patiño-Restrepo JF. La genómica: el nuevo horizonte de la medicina. Rev Colomb Cir. 2018;33:14-6. 


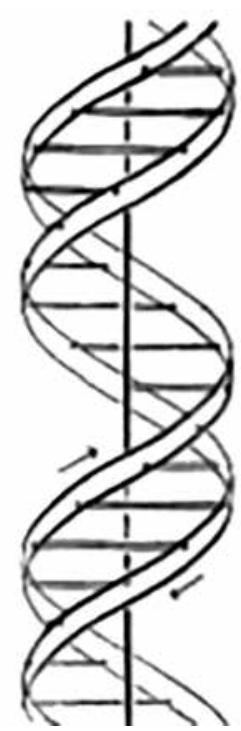

Esta es la figura que aparece en el artículo original ${ }^{1}$ de Watson y Crick en Nature en abril 25 de I953, un artículo de solo dos páginas, tal vez el más importante en la historia de la biología. También en Nature, el 30 de mayo de 1953, Watson y Crick publicaron el artículo Genetical implications of the estructure of desoxyribonucleic acid ${ }^{2}$.

En Bogotá, hace casi 40 años, tuvimos la primera reunión científica sobre el tema, un seminario convocado por la desaparecida Fundación OFA para el Avance de las Ciencias Médicas. Se publicó un libro titulado Las bases moleculares de la vida y la enfermedad, editado por J. F. Patiño Restrepo y G. Román Campos 3 .

El nuevo paradigma, el de la biomedicina, ha reinado durante todo el siglo XX; y en el siglo $\mathrm{XXI}$, con los avances en la genética y la genómica y habiendo descodificado el genoma humano ${ }^{4,5}$, otro paso trascendental en el avance de la ciencia biológica, se amplía aún más el horizonte, y la genética y la genómica aparecen como lo fundamental, el nuevo horizonte de la medicina y de la salud pública.

El National Human Genome Research Institute de los National Institutes of Health (NIH) de "an emerging medical discipline that involves using genomic information about an individual as part of their clinical care (e.g., for diagnostic or therapeutic decision-making) and the health outcomes and policy implications of that clinical use." [Una disciplina médica emergente que involucra la información genómica sobre un individuo como parte de su atención clínica (por ejemplo. para la toma de decisiones diagnósticas o terapéuticas) y los resultados o desenlaces en la atención de la salud y las implicaciones sobre políticas en tal uso clínico].

Es el nuevo campo de la medicina y, especialmente, de la cirugía personalizada, como ya aparece en la cirugía oncológica, la farmacología, las enfermedades infecciosas y las enfermedades raras.

En el campo de la cirugía oncológica, la genómica ha tenido un rápido desarrollo y permite tomar decisiones racionales en cada caso individual.

Por supuesto, el campo de la genómica en cirugía se vislumbra cada día más amplio y prometedor en cuanto a resultados o desenlaces finales.

La nueva expresión de 'medicina traslacional', ha sido definida por la European Society for Translational Medicine, como "an interdisciplinary branch of the biomedical field supported by three pillars: benchside, bedside and community" ". 'Benchside' se refiere al laboratorio de investigación, 'bedside' a la atención clínica y 'community' a la comunidad. Es el enfoque multidisciplinario y transdisciplinario de la banca del laboratorio de investigación a la cama del paciente, "del laboratorio a la cama del enfermo". Su objetivo es combinar las disciplinas, recursos, experiencia y conocimiento, y técnicas, a fin de promover resultados superiores en la prevención, el diagnóstico y la terapéutica y, con ello, mejorar la atención global de la salud.

Aparece aquí la genómica en papel preponderante al llevar a la atención personalizada. Se dice que las guías de práctica clínica, tan queridas por las EPS de nuestro desastroso Sistema General de Seguridad Social en Salud que creó la Ley IOo del 23 de diciembre de 1993, cuyo ponente fue el entonces senador Álvaro Uribe Vélez, tendrán cada vez menos aplicación individual en la medida en que la medicina se vuelve más y más personalizada, y cobra fuerza el dictum los médicos no tratamos enfermedades, tratamos enfermos. 
Algunos médicos un tanto escépticos que han mirado este movimiento de la medicina o salud traslacional con algo de ironía, deben saber que los National Institutes of Health (NIH) lo han impulsado vigorosamente, especialmente en el campo de la investigación biomédica, con énfasis en la colaboración entrecruzada entre los investigadores y los clínicos, y desde diciembre de 20II, ya existe el National Center for Advancing Translational Science (NCATS) como parte de los NIH. Varias de las grandes universidades del mundo, entre ellas las de Berkeley y Tulane, tienen programas de maestría y doctorado en salud traslacional, y el NCATS apoya económicamente a cerca de un centenar de centros de medicina traslacional en los Estados Unidos.

Propósito fundamental de la medina traslacional es llevar los resultados de la investigación científica prontamente a la práctica clínica.

Con el desarrollo de nuevas pruebas de genómica, ya comercialmente disponibles, esto será cada día más fácil y más efectivo, y la genómica adquirirá día a día mayor preponderancia en la evaluación clínica y en la terapéutica, algo de sumo valor en cirugía, por cuanto una intervención quirúrgica siempre representa un riesgo. Con las pruebas de genómica, la toma de decisiones se racionaliza y los resultados de la cirugía serán cada día mejores.
Este es el esplendoroso panorama que se vislumbra ahora para los cirujanos en ejercicio, y que representa un advenimiento de tanta importancia como lo fueron la anestesia, la antisepsia, los antibióticos, la nutrición parenteral total ${ }^{7}$ y, luego, los trasplantes de tejidos y órganos y la cirugía mínimamente invasora.

\section{Referencias}

I. Watson JD, Crick FHC. Molecular structure of nucleic acids: A structure for desoxiribonucleic acid. Nature. I953:I7I:737-8.

2. Watson JD, Crick FH. Genetical implications of the structure of desoxyribonucleic acid. Nature I953:I7I:964-7.

3. Patiño Restrepo JF, Román Campos G, editores. Las bases moleculares de la vida y de la enfermedad. Bogotá: Fundación OFA para el Avance de las Ciencias Biomédicas; 1980.

4. Patiño Restrepo JF. Genoma humano, bioética y clonación. Innovación y Ciencia (edición especial). 200I;IX:I4-25.

5. Patiño JF. Genoma humano, bioética y clonación. En: Asociación Colombiana para el Avance de la Ciencia, editores. El genoma humano. Bogotá: Panamericana Editorial Ltda.; 2002.

6. Cohrs RJ, Martin T, Ghahramani P, Bidaut L, Higgins PJ, Shahzad, A. Translational medicine definition by the European Society for Translational Medicine. New Horizons in Translational Medicine. 20I5;2:86-8. DOI: http://doi.org/IO.IOI6/j.nhtm.2014.I2.002.

7. Stevens JC. Surgical nutrition: The fourth coming. JAMA. 1978;239:192. 